\title{
Outbreak of abomasal bloat in goat kids due to Clostridium ventriculi and Clostridium perfringens type $\mathrm{A}$ in Brazil
}

\author{
Mário Felipe Balaro ${ }^{1}$, Fernanda Gonçalves ${ }^{1}$, Felipe Seabra Leal ${ }^{1}$, Isabel Cosentino ${ }^{1}$, Júlia \\ Vignoli $^{1}$, Nathalia Silva ${ }^{1}$, Felipe Brandão ${ }^{1}$, Alessandra Figueiredo Nassar ${ }^{2}$, Simone $^{2}$ \\ Miyashiro $^{2}$, Nathalie Cunha ${ }^{1}$, and Claudia Del Fava ${ }^{2}$ \\ ${ }^{1}$ Universidade Federal Fluminense \\ ${ }^{2}$ Instituto Biologico
}

May 23, 2022

\begin{abstract}
This study aimed to describe an outbreak of abomasal bloat in kid goats and its clinical, pathological, microbiological, molecular, and epidemiological characteristics. In the kidding season, increased mortality of kid goats with a history of abdominal bloating, dullness, and death was reported. Clinical examinations were carried out, and biological samples from necropsied kids $(\mathrm{n}=11)$ were collected for pathological, microbiological, and molecular diagnosis. Likewise, an epidemiological survey was carried out in order to verify possible associated factors related to the disorder. A therapeutic protocol was also implemented. The main necropsy findings were dehydration, pale mucosa, ascites, abomasal and intestinal meteorism and congestion, emphysematous abomasitis, and cranial areas of lung consolidation. Through staining techniques for cytological evaluations of the abomasum, it was possible to identify Gram positive bacteria, coccoid, with a cuboid shape suggestive of Clostridium ventriculi, Gram positive bacilli suggestive of Clostridium perfringens and ovoid basophilic yeasts compatible with Saccharomyces cerevisiae. By anaerobic culture and molecular tests, C. ventriculi and C. perfringens type A were confirmed. The main histopathological findings were cholangiohepatitis, nephrosis, emphysematous abomasitis, hyalinization of the gastric and intestinal walls, gastroenteritis, intestinal thromboembolism, pulmonary edema, and non-purulent pneumonia, overall suggesting a systemic enterotoxemia picture. The early detection of sick kids and quick initiation of treatment were the primary determinants of the prognosis of each case. There was a final mortality rate of $24.4 \%$ (20/82), and the agents C. perfringens type A and C. ventriculi were identified as the main ones involved, with the possible participation of S. cerevisiae. Among the possible associated factors, the erroneous use of the milk replacer associated with inadequate kid management was verified. Among the prophylactic measures, hygiene care, proper use of milk replacer, vaccination plan containing C. perfringens alpha toxoid associated with a good colostrum management were suggested.
\end{abstract}

Outbreak of abomasal bloat in goat kids due to Clostridium ventriculi and Clostridium perfringens type A in Brazil

Outbreak of abomasal bloat in goat kids

Mario Felipe Alvarez Balaro ${ }^{1 *}$; Fernanda Martins Gonçalves ${ }^{1}$; Felipe Seabra Cardoso Leal ${ }^{1}$; Isabel Oliveira Cosentino $^{1}$; Júlia Alves Vignoli ${ }^{2}$, Nathalia Xavier da Silva ${ }^{2}$; Felipe Zandonadi Brandão ${ }^{1}$; Alessandra Figueiredo de Castro Nassar ${ }^{3}$; Simone Miyashiro ${ }^{3}$ N Nathalie Costa da $\mathrm{Cunha}^{2}$; Claudia Del Fava ${ }^{4}$.

${ }^{1}$ Department of Pathology and Clinical Veterinary, Faculty of Veterinary, Fluminense Federal University, Rua Vital Brasil Filho, 64, zip code: 24320-340, Niteroi, RJ, Brazil.

${ }^{2}$ Department of Veterinary Collective Health and Public Health, Faculty of Veterinary, Fluminense Federal University, Rua Vital Brasil Filho, 64, zip code: 24320-340, Niteroi, RJ, Brazil. 
${ }^{3}$ General Bacteriology Laboratory, Biological Institute, São Paulo, SP, Brazil.

${ }^{4}$ Pathological Anatomy Laboratory, Biological Institute, São Paulo, SP, Brazil.

${ }^{*}$ Corresponding author: Department of Pathology and Clinical Veterinary, Faculty of Veterinary, Fluminense Federal University, Rua Vital Brasil Filho, 64, zip code: 24320-340, Niteroi, RJ, Brazil. E-mail address: mariobalaro@id.uff.br (MFA Balaro).

\section{SUMMARY}

This study aimed to describe an outbreak of abomasal bloat in kid goats and its clinical, pathological, microbiological, molecular, and epidemiological characteristics. In the kidding season, increased mortality of kid goats with a history of abdominal bloating, dullness, and death was reported. Clinical examinations were carried out, and biological samples from necropsied kids $(\mathrm{n}=11)$ were collected for pathological, microbiological, and molecular diagnosis. Likewise, an epidemiological survey was carried out in order to verify possible associated factors related to the disorder. A therapeutic protocol was also implemented. The main necropsy findings were dehydration, pale mucosa, ascites, abomasal and intestinal meteorism and congestion, emphysematous abomasitis, and cranial areas of lung consolidation. Through staining techniques for cytological evaluations of the abomasum, it was possible to identify Gram positive bacteria, coccoid, with a cuboid shape suggestive of Clostridium ventriculi, Gram positive bacilli suggestive of Clostridium perfringens and ovoid basophilic yeasts compatible with Saccharomyces cerevisiae. By anaerobic culture and molecular tests, C. ventriculi and C. perfringens type A were confirmed. The main histopathological findings were cholangiohepatitis, nephrosis, emphysematous abomasitis, hyalinization of the gastric and intestinal walls, gastroenteritis, intestinal thromboembolism, pulmonary edema, and non-purulent pneumonia, overall suggesting a systemic enterotoxemia picture. The early detection of sick kids and quick initiation of treatment were the primary determinants of the prognosis of each case. There was a final mortality rate of $24.4 \%$ (20/82), and the agents $C$. perfringens type $\mathrm{A}$ and $C$. ventriculi were identified as the main ones involved, with the possible participation of $S$. cerevisiae. Among the possible associated factors, the erroneous use of the milk replacer associated with inadequate kid management was verified. Among the prophylactic measures, hygiene care, proper use of milk replacer, vaccination plan containing $C$. perfringens alpha toxoid associated with a good colostrum management were suggested.

KEYWORDS: Artificial feeding, goats, Clostridium spp., enterotoxemia, milk replacement. 